\title{
心理咨询理论与实践
}

\section{肯定性咨询法：产生背景、理论 基破与学校实践}

\author{
郭材欣 \\ 湖南人文科技学院教育学院, 娄底, 417000 \\ 邮箱: 2647123246@qq.com
}

摘 要: 性少数人群包括女同性恋、男同性恋、双性恋和跨性别者, 性少数个 体受到更多来源的压力, 更需要心理咨询的帮助。肯定性咨询法对于性少数的 心理咨询更具文化上的适当性，是在传统心理咨询理论与其他相关理论结合的 基础上建构起来的。肯定性咨询法运用于学校实践对于师生的心理健康、良好 校园文化的建构、性少数群体的学校心理咨询具有积极意义。培养学校肯定性 咨询法咨询师要注意对不同性取向和性别认同的态度和信念、获取性少数群体 的相关知识、理解学校心理咨询的特殊性。肯定性咨询法的学校实践还要关注 培训和咨询效果评估、中国文化背景、学校心理咨询的独立性建设和融入校园 活动等方面。

关键词: 肯定性咨询法; 性少数; 学校心理咨询; 心理咨询师

收稿日期：2019-06-21；录用日期：2019-07-13；发表日期：2019-07-28 


\title{
Affirmative psychotherapy and counseling: Background, Theoretical Basis and School Practice
}

\author{
Guo Caixin \\ School of Education, Hunan University of Humanities, Science and Technology,
}

Loudi 417000

Abstract: Sexual minorities, including lesbian, gay, bisexual and transgender, are under pressure from more sources and need counseling. Affirmative psychotherapy and counseling is more culturally appropriate for psychological counseling for sexual minorities, and is constructed on the basis of the combination of traditional psychological counseling theory and other related theories. The application of affirmative psychotherapy and counseling to school practice is of positive significance to the mental health of teachers and students, the construction of excellent campus culture and the school counseling of sexual minorities. To train LGBT-affirmative counselors in schools should pay attention to attitudes and beliefs about different sexual orientations and gender identities, acquire relevant knowledge of sexual minorities, and understand the particularity of school counseling. The school practice of affirmative psychotherapy and counseling should also pay attention to the evaluation of training and consultation effect, the Chinese cultural background, the independent construction of the school counseling and the integration of campus activities.

Key words: Affirmative psychotherapy and counseling; Sexual minority; School counseling; Counselor

Received: 2019-06-21; Accepted: 2019-07-13; Published: 2019-07-28 
Copyright (C) 2019 by author(s) and SciScan Publishing Limited

This article is licensed under a Creative Commons Attribution-NonCommercial 4.0 International License.

https://creativecommons.org/licenses/by-nc/4.0/

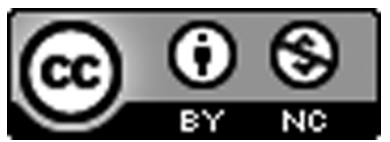

\section{1 肯定性咨询法的产生背景}

性少数（Sexual Minority）是指性取向为异性恋之外的少数性取向人群 [1]，包 括性取向为同性恋（女同性恋 Lesbian 和男同性恋 Gay）、双性恋（Bisexual）以及 性别认同为跨性别（Transgender）的群体 [2], 简称为 LGBT。研究表明, LGBT 群 体的心理健康状况与异性恋相比更为消极，导致更多的抑郁、焦虑、物质滥用 [3][4] [5][6]，有着更高水平的终身自杀意念和的终身自杀未遂率 [3][7][8][9]。

根据性少数压力模型（Sexual Minority Stress Model），影响 LGBT 群体心理健 康状况的压力包括远端压力（一般压力、外部性少数压力 ) 和近端压力（内部性 少数压力 ) $[1][10]$ 。一方面, 社会环境中存在的一般压力（个体的工作状况和社会 经济地位等) 和由于性少数压力相关的偏见事件 (歧视、暴力等) 等远端压力可 以直接影响个体的心理健康水平; 另一方面，一般压力和远端压力也可以内化为 恐同、恐跨、害怕身份暴露与拒绝等近端压力，间接影响个体的心理健康水平。 有的研究涉及了 LGBT 群体遭受的歧视和侵害等远端压力，例如更容易遭受到人 身攻击和性侵害 [11][12]，80\% 以上的跨性别人群遭受到了与他们的性别认同有关 的口头辱骂 [13]; 也有不少研究探讨了 LGBT 群体内化的近端压力, 例如内化的 对自己性别认同的差耻感和消极情绪 [14][15]，努力对他人隐瞒性取向和性别认同 [16][17]。

由于性少数人群面对更多来源的压力，更容易发生心理健康问题 [18], 因此 他们更迫切需要心理健康服务, 心理咨询就是其中之一。但是, 一方面, 部分心 理咨询的有关从业人员对 LGBT 群体的相关知识较为缺乏，甚至采用以异性恋和 
顺性别为目标的心理学疗法 [19][20][21], 存在异性恋主义和顺性别社会规范背景 下的偏见 [22]; 另一方面，LGBT 群体自身存在着对暴露个人性取向或性别认同的 后果的担忧 [21]。这些原因导致了 LGBT 群体对当前心理咨询服务的不满或者不 接受。因此，为 LGBT 人群提供在文化上适当的和更加肯定其性别认同的心理咨 询是必要的 [23]。肯定性咨询法就是在这一背景下逐渐发展起来的 [18]。

\section{2 肯定性咨询法的理论基础}

肯定性咨询法（Affirmative Psychotherapy and Counseling）是一种鼓励非异性恋 或非顺性别的来访者接受其性倾向、性别认同和性别表达，而非试图将非异性恋 者或非顺性别者扭转为 “正常人”，或劝说其减少甚至消除同性恋、跨性别欲望 或行为的心理疗法 [24]。肯定性咨询法不是一种独立的心理咨询理论取向或技术， 而是在咨询师现有的咨询理论取向上增加对 LGBT 群体肯定的态度和理念、相关 的知识和技能 [23][25], 强调性少数不是心理疾病，同性恋、双性恋和跨性别身份 和异性恋身份一样，都是积极的人类生活经验和表达方式，个体接受并肯定自己 是其从心理不适中恢复过来的关键 $[25]$ 。

肯定性咨询法是在传统心理咨询理论与其他相关理论结合基础上建构起来的 [24]，这些相关理论包括女权主义理论、性人权理论、酷儿理论和多元文化咨询理论。 女权主义理论认为，个人的经验与社会政治现实有关，因此，来访者的心理痛苦 不应该被当作缺陷的证据，而应当看到痛苦背后对不公正制度的抵抗。咨询师不 仅要促进来访者的个人成长，还要推进社会的变化 [24][26]。此外，女权主义理论 还提倡咨询师与来访者建立平等的咨询关系，提倡多元方法论 [26], 克服性别偏 见 [27]。性人权理论认为, 性权利是人之作为性存在所有和应当享有的人权，而 与其他身份无关，因此，应从人权的角度看待性的多样性 [24][28]，把性少数视为 一项基本人权, 反对心理咨询把性少数作为 “心理问题” 对待 [24]。酷儿理论认为, 身份是动态的和变化的，性别是被历史和社会所建构的，性倾向可以在无意识的 情况下发生自发的改变 [24]，因此，咨询师不应该借助异性恋的权利对其他群体 进行 “正规化”，而应该帮助性少数来访者自由地、勇敢地接受自己的意愿性别 或性倾向。多元文化咨询理论认为，咨询师要重视并认同文化变量交互作用对个 
体的影响和个体文化身份的流动性和复杂性 [24][29]，因此，咨询师应该了解性的 多元性, 学习多元文化的知识和技能,帮助性少数来访者消除社会环境导致的恐同、 恐跨等压力。

\section{3 肯定性咨询法的学校实践}

\section{1 肯定性咨询法在学校实践中的意义}

\subsection{1 提高师生的心理健康水平}

根据性少数压力模型, 学校中的 LGBT 群体不仅受到学习工作状况、家庭经 济和社会地位等一般压力, 还会受到学校师生的歧视和暴力等外部性少数压力 [1] [10]。研究表明, 性少数的学生更容易遭受校园欺凌, 受到同学的口头辱骂、肢体 攻击和威胁 [30][31][32][33][34][35], 此外, 我国的性少数群体由于 “家本位” 的文 化背景面临更大的家庭压力 [36][37], 这样的环境使得学校中的 LGBT 群体难以被 老师、同学和父母接受，从而内化为恐同、恐跨、害怕身份暴露与拒绝等内部压 力 [10]。学校心理咨询室的咨询师如果接受了性少数来访者, 又不能以性少数的 肯定性立场为师生提供咨询服务，就有可能进一步损害来访者的自尊 [24]。因此， 对于学校心理咨询, 咨询师采用传统心理咨询中倡导的中立态度也不足以解除性 少数来访者身上的 “枷锁” ，只有遵循不假定来访者是异性恋、不试图探讨来访 者性倾向的成因、帮助来访者减少内化的恐惧并完成自我认同等基本原则 [38], 才能够避免伤害来访者。学校心理咨询采用肯定性咨询法有利于性少数来访者在 学校中寻求到情感支持, 提高自尊和自我决定水平, 获得更多学习和了解自己的 途径, 从而更好地完成自我认同。综上, 肯定性咨询法在学校实践有助于提高学 校师生的心理健康水平。

\subsection{2 建构更加和谐、开放、进步的校园文化}

根据女权主义理论和多元文化咨询理论，肯定性咨询法为学校内部性别刻板 印象的消除、多元文化的整合和生生之间、师生之间、师师之间的心理相容提供 了参考价值 [24][26][27][29]。首先，肯定性咨询法提倡咨询师不仅要促进个体的成 长, 还要推进社会的变革 [24][26], 因此, 学校心理咨询室在改善学校内部由于异 
性恋主义和顺性别主义导致的暴力、歧视和恐惧上扮演着重要的角色; 其次，研 究表明，对变化持开放态度、更加自我超越、更少保守主义的价值取向的个体对 同性恋者的态度更加积极 [39]，从反面来说，对性少数者持歧视、偏见和恐惧的 校园文化则不利于师生的进步和自我超越；最后，肯定性咨询法使全校师生处于 一个具有安全感、归属感和被接纳的环境中，有助于建构更具人文关怀、更加和 谐的校园文化。尊重个体的自主选择和人性意愿，就是尊重人本身，这关乎到每 一个社会人的和谐发展。综上, 肯定性咨询法在学校实践有助于建构更加和谐、 开放、进步的校园文化。

\section{1 .3 解决学校心理咨询针对性少数群体的咨询伦理问题}

传统心理咨询认为同性恋或跨性别是 “病理性” 的，试图改变来访者的性倾 向或性行为，我国的心理咨询虽然没有明确 “改变性倾向或性行为” 的目的，但 实际上却在谨慎地引导性少数者变为异性恋者 [24]。这些错误的观念或方法包含 着社会偏见，尤其是在学校心理咨询中，咨询师的偏见会给来访者造成伤害，甚 至导致来访者对学校失去希望, 产生自卑、厌学等不良情绪。咨询中试图扭转来 访者的性倾向甚至是违反伦理原则的，在这一过程中，咨询师没有向来访者提供 性倾向是否可以扭转的科学证据, 并且给同性恋者和跨性别者贴上不幸福、错误的、 悲惨的标签，违背了诚信的伦理原则 [24]; 本身对性少数持有刻板印象和偏见的 咨询师在缺乏性少数群体相关知识和技能的基础上接受性少数来访者的咨询，违 背了具备能力这一伦理原则 [39]。肯定性咨询法强调咨询师掌握多元文化的知识, 从而更好地帮助来访者协调好性倾向和性行为与家庭、社区、其他信念体系的关系， 完成自我认同，遵守了咨询的基本伦理原则和职业道德。

\section{2 学校肯定性咨询法咨询师的培养}

由于肯定性咨询法在学校实践中具有诸多积极意义, 而许多心理服务工作者 认为自身缺乏与性少数群体建立合作关系的知识和技能 [40][41], 因此, 学校对肯 定性咨询法咨询师的培养显得尤为重要。培养学校肯定性咨询法咨询师需要注意 以下问题:

第一，检查咨询师对自身和他人的性取向和性别认同的态度和信念。一方面， 
咨询师要认识到自身对性少数的先前偏见和错误信念会阻碍其接受肯定性咨询法 的信念 [25]。另一方面, 咨询师要探索自身存在哪些阻碍接受肯定性咨询法信念 的异性恋假设和异性恋特权，方刚和杨志红 [24] 认为肯定性咨询法咨询师可从 3 个步骤进行自我探索：（1）探索异性恋假设，反思自身无意识的异性恋假设的形 成过程及其对咨询的影响;（2）探索异性恋特权，反思异性恋特权给异性恋者带 来了哪些利益, 认识到异性恋特权的存在及其对性少数群体造成的消极影响;（3 ) 探索异性恋身份的发展，异性恋咨询师要反思自己是如何成为异性恋的，这有助 于咨询师意识到自己的性倾向会如何影响咨询过程。

第二，获得性少数的压力类型、心理健康状况、独特临床问题、文化以及生 活方式等相关知识。要消除咨询师对性少数的先前偏见和错误信念, 从而更好地 接受肯定性咨询法, 首先应该深人了解性少数群体内部的多元性和不同的生活方 式，掌握性别认同和性倾向的相关知识，熟悉性少数群体面对的压力类型 [24], 全面掌握有关信息是消除偏见的基础; 其次，咨询师要了解性少数群体的身份发 展问题, 包括这一过程中所涉及的心理和社会因素 [23][42], 从而更好地判断性少 数来访者当前问题的形成原因; 最后, 了解不同性少数人群的独特问题和经历, 比如有宗教背景的性少数的独特问题、同性恋和双性恋的特点和独特经历、跨性 别者面对的困难和处境。了解这些特定的问题，以及与这些不同的来访者建立咨 询关系的技能，对于给性少数提供有效的心理咨询至关重要。

第三，根据学校心理咨询的特殊性实施肯定性咨询法。首先，要意识到并避 免双重关系对咨询的消极影响。在学校心理咨询中, 咨询师往往还同时具备老师 或管理者的身份，难以避免地产生双重关系 [43]，咨询师在无法避免双重关系产 生时，应努力减少双重关系对咨询的消极影响，比如减少教师和管理者的权威， 赋权给学生, 给学生更多自主选择的机会, 保障学生的利益, 注意双重关系对自 己判断的影响, 避免刻板印象阻碍学生的求助意愿 [44]; 其次, 要避免学生家长 或其老师对咨询目标和咨询关系的影响。在学校心理咨询中, 有些性少数群体可 能是被其家长或老师带来矫正的，咨询师不应该根据学生家长或其老师的意愿来 进行咨询 [24], 而应该告知他们改变性倾向是违反伦理原则和职业道德的，甚至 有虐待儿童之嫌 [24][45]; 最后, 要在帮助性少数学生减少内部压力的同时, 减少 
学生的外部压力。学校肯定性咨询法咨询师可以通过讲座、宣传、与教师和管理 者沟通、培训肯定性咨询法咨询师的方式来减少学校环境对性少数群体施加的压 力。此外, 高校的心理学专业课程中应该增设肯定性咨询法, 这有助于培养更多 对性少数群体持有正确态度的心理学专业工作者 [24]。

\section{3 肯定性咨询法在学校实践中的展望}

外部的偏见和暴力以及内部的恐同、恐跨情绪严重损害着性少数群体的身心 健康，学校作为一个提供科学知识的场所，对于社会关于性少数态度的走向起着 重要的引导作用，对于性少数个体的自我认同也起着关键作用。因此，对肯定性 咨询法在学校实践的讨论具有重要的意义，不仅可以提高师生的心理健康水平， 同时也有助于建构更加和谐、开放、进步的校园文化，为性少数群体的学校心理 咨询提供了新视角。本文还初步探讨了学校肯定性咨询法咨询师的培养问题，今 后对于肯定性咨询法的学校实践还可以从以下几个方面着手。

第一，学校肯定性咨询法咨询师的培训和咨询效果评估。目前，肯定性咨询 法在我国还没有系统的培训,在学校中应用肯定性咨询法必然会遇到更多的问题, 例如家长和学校老师的不理解、来访者心理问题的复杂性和双重关系及保密问题。 因此, 研究者和学校心理咨询工作者都要加大普及力度, 完成社会对学校应用肯 定性咨询法的了解、接受和认同，纳人更多社会工作者进行培训。相关研究者还 要参与到学校实践中去, 考察肯定性咨询法的培训效果以及个体差异性, 运用咨 询实践及时评估肯定性咨询法的咨询效果。

第二，探讨中国文化背景下肯定性咨询法的实践问题。性少数群体的心理健 康与文化背景密切相关，社会生态学视角强调探讨社会文化对同性恋者心理健康 的作用 [46]，女权主义理论强调来访者的心理痛苦与不公正的社会制度有关。研 究表明，我国同性恋者受到中国 “家本位” 的影响，相较于个性解放更看重家庭 和谐，从而面临更大的家庭压力 [36][37]，此外，Hofstede 的研究表明我国对男子 气概的偏好远高于部分北欧国家 [47], 这与我国的主流男权文化密切相关，这使 得不符合男子气概的男同性恋或跨性别者遭受到更多的攻击和污名。肯定性咨询 法不是以客观中立的态度对待性少数, 而是以积极肯定的态度对待性少数的性倾 
向和性行为，这种旗帜鲜明的肯定容易使来访者或反对者认知失调，因此，如何 在肯定的同时保持理性和避免他人对咨询师的不信任是肯定性咨询法本土化实践 时需要探讨的问题。

第三，加强学校心理咨询的独立性建设。由于学校心理咨询中的咨询师往往 具有多重角色，这可能会导致来访者的顾虑 [44]，对于性少数来访者尤其如此， 因此，今后可以探讨学校心理咨询工作的独立性建设途径，最大限度地减少多重 关系现象的出现。

第四，在校园活动中融人肯定性咨询法的有关理念。比如，在防艾活动中， 不对同性恋错误地贴上艾滋病高危人群的标签，而是在普及 “如何做好防范” 的 同时宣传多样的性倾向和性别选择都是值得肯定的。也可以在组织社团活动的同 时融人倡导性的多样性的心理健康教育、性教育和多元文化教育。目前, 在学校中, 专门针对性少数群体的维权与心理健康建设的活动还比较少, 今后可以探讨在校 园活动中融人肯定性咨询法相关理念的途径。

\section{参考文献}

[1] 张静, 郑丽军, 郑涌. 性少数人群的心理健康: 理论模型与研究取向 $[\mathrm{J}]$. 心理科学进展, 2015, 23 ( 6 ) : 1021-1030.

[2] 魏重政, 刘文利. 性少数学生心理健康与遭受校园欺凌之间关系研究 $[\mathrm{J}]$. 中国临床心理学杂志，2015，23 (4)：701-705.

[3] King M, Semlyen J, Tai S S, et al. A systematic review of mental disorder, suicide, and deliberate self harm in lesbian, gay and bisexual people $[\mathrm{J}]$. BMC psychiatry, 2008, 8 ( 1 ) : 70 .

https://doi.org/10.1186/1471-244X-8-70

[4] Bockting W O, Miner M H, Swinburne R E, et al. Stigma, mental health, and resilience in an online sample of the US transgender population [ $\mathrm{J}]$. American journal of public health, 2013, 103 ( 5 ) : 943-951.

https://doi.org/10.2105/AJPH.2013.301241

[ 5 ] Leonard W, Lyons A, Bariola E. A closer look at private lives 2: addressing 
the mental health and wellbeing of lesbian, gay, bisexual, and transgender ( LGBT ) Australians [ J $] .2015$.

[6] Lea T, de Wit J, Reynolds R. Minority stress in lesbian, gay, and bisexual young adults in Australia: Associations with psychological distress, suicidality, and substance use [ J ] . Archives of sexual behavior, 2014, 43 ( 8 ): 1571-1578. https://doi.org/10.1007/s10508-014-0266-6

[7] Grossman A H, D'Augelli A R. Transgender youth and life threatening behaviors [J ] . Suicide and Life Threatening Behavior, 2007, 37 ( 5) : 527-537. https://doi.org/10.1521/suli.2007.37.5.527

[8] Mathy R M. Suicidality and sexual orientation in five continents: Asia, Australia, Europe, North America, and South America [ J ] . International Journal of Sexuality and Gender Studies, 2002, 7 ( 2-3 ) : 215-225. https://doi.org/10.1023/A:1015853302054

[9] Plöderl M, Sellmeier M, Fartacek C, et al. Explaining the suicide risk of sexual minority individuals by contrasting the minority stress model with suicide models $[\mathrm{J}]$. Archives of sexual behavior, 2014, 43 ( 8 ) : 1559-1570. https://doi.org/10.1007/s10508-014-0268-4

[10 ] Meyer I H. Prejudice, social stress, and mental health in lesbian, gay, and bisexual populations: conceptual issues and research evidence $[\mathrm{J}]$. Psychological bulletin, 2003, 129 ( 5 ) : 674.

https://doi.org/10.1037/0033-2909.129.5.674

[11] Katz-Wise S L, Hyde J S. Victimization experiences of lesbian, gay, and bisexual individuals: A meta-analysis $[\mathrm{J}]$. Journal of sex research, 2012, 49 ( 2-3 ) : 142-167. https://doi.org/10.1080/00224499.2011.637247

[12 ] Stotzer R L. Violence against transgender people: A review of United States data $[\mathrm{J}]$.Aggression and Violent Behavior, 2009, 14 ( 3 ) : 170-179. https://doi.org/10.1016/j.avb.2009.01.006

[13 ] Clements-Nolle K, Marx R, Katz M. Attempted suicide among transgender 
persons: The influence of gender-based discrimination and victimization $[\mathrm{J}]$.

Journal of homosexuality, 2006, 51 ( 3 ) : 53-69.

https://doi.org/10.1300/J082v51n03_04

[ 14 ] Hendricks M L, Testa R J. A conceptual framework for clinical work with transgender and gender nonconforming clients: An adaptation of the Minority Stress Model[ J ]. Professional Psychology: Research and Practice, 2012, 43( 5 ): 460. https://doi.org/10.1037/a0029597

[ 15 ] Newcomb M E, Mustanski B. Internalized homophobia and internalizing mental health problems: A meta-analytic review $[\mathrm{J}]$. Clinical psychology review, 2010, 30 ( 8 ) : 1019-1029. https://doi.org/10.1016/j.cpr.2010.07.003

[16] Jackson S D, Mohr J J. Conceptualizing the closet: Differentiating stigma concealment and nondisclosure processes $[\mathrm{J}]$. Psychology of Sexual Orientation and Gender Diversity, 2016, 3 ( 1 ) : 80 .

https://doi.org/10.1037/sgd0000147

[17] Pachankis J E. The psychological implications of concealing a stigma: A cognitive-affective-behavioral model $[\mathrm{J}$ ] . Psychological bulletin, 2007, 133 ( 2 ) : 328. https://doi.org/10.1037/0033-2909.133.2.328

[18 ] King M, Semlyen J, Killaspy H, et al. A systematic review of research on counselling and psychotherapy for lesbian, gay, bisexual \& transgender people[ $\mathrm{J}]$. British Association for Counselling and Psychotherapy, 2007.

[ 19] Palma T V, Stanley J L. Effective counseling with lesbian, gay, and bisexual clients [ J ] . Journal of College Counseling, 2002, 5 ( 1 ) : 74-89. https://doi.org/10.1002/j.2161-1882.2002.tb00208.x

[20 ] Pepping C A, Lyons A, Halford W K, et al. Couple interventions for samesex couples: A consumer survey $[\mathrm{J}]$. Couple and Family Psychology: Research and Practice, 2017, 6 ( 4 ) : 258.

https://doi.org/10.1037/cfp0000092

[21 ] Rispel L C, Metcalf C A, Cloete A, et al. You become afraid to tell them that 
you are gay: health service utilization by men who have sex with men in South African cities $[\mathrm{J}]$. Journal of public health policy, 2011, 32 ( 1 ) : 137151. https://doi.org/10.1057/jphp.2011.29

[22] Meyer I H. Minority stress and mental health in gay men [ J ] . Journal of health and social behavior, 1995: 38-56. https://doi.org/10.2307/2137286

[23 ] Pachankis J E, Goldfried M R. Clinical issues in working with lesbian, gay, and bisexual clients $[\mathrm{J}]$. Psychotherapy: Theory, research, practice, training, 2004, 41 ( 3 ) : 227. https://doi.org/10.1037/0033-3204.41.3.227

[24 ] 方刚, 杨志红. 肯定性咨询法 [ M ] . 北京：中国社会科学出版社, 2015 .

[25] Davies D. Towards a model of gay affirmative therapy [ M ] . Buckingham, England: Open University Press. 1996.

[26] 郭爱妹. 论女性主义心理治疗观 $[\mathrm{J}$ ] . 南京师大学报（社会科学版）, 2006 ( 6 ) : 108-113.

[27] Weisstein N. Psychology constructs the female; or the fantasy life of the male psychologist ( with some attention to the fantasies of his friends, the male biologist and the male anthropologist ) [ J ] . Feminism \& Psychology, 1993, 3 ( 2 ) : 194-210. https://doi.org/10.1177/0959353593032005

[28］赵合俊。“性权利” 应率先进人 “妇女法” 一一种理论的说明 $[\mathrm{J}]$. 中华女子学院学报, 2003, 15 ( 5 ) : 1-5.

[29] Constantine M G. Predictors of satisfaction with counseling: Racial and ethnic minority clients' attitudes toward counseling and ratings of their counselors' general and multicultural counseling competence $[\mathrm{J}]$. Journal of Counseling Psychology, 2002, 49 ( 2 ) : 255.

https://doi.org/10.1037//0022-0167.49.2.255

[30 ] Berlan E D, Corliss H L, Field A E, et al. 22: Sexual orientation and bullying in adolescents $[\mathrm{J}]$. Journal of Adolescent Health，2007，40 ( 2): S28. https://doi.org/10.1016/j.jadohealth.2006.11.075

[31 ] Berlan E D, Corliss H L, Field A E, et al. Sexual orientation and bullying 
among adolescents in the growing up today study $[\mathrm{J}]$. Journal of Adolescent Health, 2010, 46( 4 ): 366-371. https://doi.org/10.1016/j.jadohealth.2009.10.015

[32] Russell S T, Ryan C, Toomey R B, et al. Lesbian, gay, bisexual, and transgender adolescent school victimization: Implications for young adult health and adjustment $[\mathrm{J}]$. Journal of School Health, 2011, 81 ( 5 ) : 223-230. https://doi.org/10.1111/j.1746-1561.2011.00583.x

[33 ] Olsen E O M, Kann L, Vivolo-Kantor A, et al. School violence and bullying among sexual minority high school students, 2009-2011 [ J ] . Journal of Adolescent Health，2014，55 ( 3 ) : 432-438.

https://doi.org/10.1016/j.jadohealth.2014.03.002

[ 34 ] Saewyc E M, Skay C L, Pettingell S L, et al. Hazards of stigma: the sexual and physical abuse of gay, lesbian, and bisexual adolescents in the United States and Canada [ J ] . Child welfare, 2006, 85 ( 2 ) : 195-213.

[35 ] 彭畅, 黄云香, 刘小群, 等. 高中生性取向与校园欺负行为关系 $[\mathrm{J}]$. 中国公共卫生，2019：1-4.

[36 ] 景军, 王晨阳, 张玉萍. 同性恋的出柜与家本位的纠结 $[\mathrm{J}]$. 青年研究, 2014 ( 5 ) : 79-86.

[ 37 ] Wah-Shan C. Homosexuality and the cultural politics of Tongzhi in Chinese societies $[\mathrm{J}]$. Journal of homosexuality, 2001, 40 ( 3-4 ) : 27-46. https://doi.org/10.1300/J082v40n03_03

[ 38 ] Appleby G A, Anastas J W. Not just a passing phase: Social work with gay, lesbian, and bisexual people [ M ] . New York: Columbia University Press, 1998.

[39 ] Licciardello O, Castiglione C, Rampullo A. Intergroup contact, value system and the representation of homosexuality $[\mathrm{J}]$. Procedia-Social and Behavioral Sciences, 2011, 30: 1467-1471. https://doi.org/10.1016/j.sbspro.2011.10.284

[ 40 ] Jenkins D, Johnston L B. Unethical treatment of gay and lesbian people with conversion therapy $[\mathrm{J}]$. Families in Society, 2004, 85 ( 4 ) : 557-561. https://doi.org/10.1177/104438940408500414 
[41 ] Graham S R, Carney J S, Kluck A S. Perceived competency in working with LGB clients : Where are we now? [ J ] . Counselor Education and Supervision, 2012, 51 ( 1 ) : 2-16. https://doi.org/10.1002/j.1556-6978.2012.00001.x

[ 42 ] Rutherford K, McIntyre J, Daley A, et al. Development of expertise in mental health service provision for lesbian, gay, bisexual and transgender communities $[\mathrm{J}]$. Medical education, 2012, 46 ( 9 ) : 903-913. https://doi.org/10.1111/j.1365-2923.2012.04272.x

[ 43 ] Bockting W O, Coleman E. Developmental stages of the transgender coming out process: Toward an integrated identity $[\mathrm{J}]$. Principles of transgender medicine and surgery, 2007, 1: 185-208.

[44]曹宁宁,石惠,卢丽琼. 高校心理咨询师咨询伦理的现状分析与对策思考— 以上海高校为例 $[\mathrm{J}$ ] . 思想理论教育，2016（3）：80-85.

\section{https://doi.org/10.1002/stab.201690006}

[45]何元庆, 王静㜆. 高校心理咨询中非性双重关系的利弊分析与应对举措 $[\mathrm{J}$ ]. 中国临床心理学杂志，2016，24（2）：373-375.

[46] Hicks D. The importance of specialized treatment programs for lesbian and gay patients $[\mathrm{J}]$. Journal of Gay \& Lesbian Psychotherapy, 2000, 3 ( 3-4) : 81-94. https://doi.org/10.1300/J236v03n03_07

[47] 张严文, 叶宝娟. 父母拒绝教养方式对中国同性恋者自杀的影响：歧视 知觉的中介效应与出柜的调节效应 $[\mathrm{J}]$ 。 心理科学，2019，42（1）: 109-115.

[ 48 ] Hofstede G, Hofstede G J, Minkov M. Cultures and organizations: Software of the $\operatorname{mind}[\mathrm{M}]$. New York: Mcgraw-hill, 2005. 